\title{
DEVELOPMENT OF PLANT FACTORY PRODUCTION SYSTEM USING CARBON DIOXIDE AND IT-BASED INTEGRATED CONTROL SYSTEM
}

\author{
Dong Oun Lee \\ Professor, Department of Architectural \& Civil Engineering, \\ Dong-seo University, Korean \\ ldu21@hanmail.net
}

\begin{abstract}
Recently the need for plant factory is increasing in Korea and abroad in order to increase the amount of crop production throughout the year, to minimize the impact of rapidly changing environmental changes, to prefer high-quality foods by the pesticide-free method, and to supply agricultural products with low weather dependence. Interest in plant factory is increasing, but commercial production systems are still in their infancy. Since there is no separate control system to maintain the appropriate carbon dioxide concentration to maximize plant photosynthesis. Therefore, the purpose of this study is to ensure self-sufficiency of fresh vegetables without pesticides and chemical fertilizers throughout the year. For this purpose, an IT-based integrated management system is developed to build a plant factory production automation model. Also, using the oxygen produced through photosynthesis of plants and carbon dioxide produced by plant breathing, we study a continuous circulation system capable of supplying carbon dioxide for plant photosynthesis while fresh oxygen free of contaminants is supplied to the internal space.
\end{abstract}

Keywords - Plant Factory, Integrated IT Management, Carbon Dioxide, Automatic Control, Agricultural Products

\section{INTRODUCTION}

The occurrence of abnormal climate due to global warming is shifting the cultivated area and causing pests to reduce the yield, which leads to a sudden increase in the price of crops and a decrease in food self-sufficiency. In order to cope with these changes, plant factories centered on green business and organic farming are emerging as new alternatives to agricultural production. A plant factory is a system that grows crops constantly regardless of season in an artificial environment, and grows crops in a space where light, temperature, humidity, carbon dioxide, air flow, nutrients, and moisture are controlled. There are two types of plant factory: solar plant factories and fully enclosed plant factories. The biggest advantage of a plant factory is that it is possible to grow crops to meet the needs of consumers and to improve the quality of crops. In particular, it has become an active alternative to the reduction of agricultural output due to global warming.

However, optimized crop production is not achieved due to the simultaneous operation of various plants and the poor understanding of plant photosynthesis [1]. This Plant factory is a facility that can be planned year-round production by systematically 
controlling the growth environment within a controlled facility, but has low introduction cases due to high initial investment cost and maintenance cost. In addition, the currently introduced plant factories are not standardized in the basic performance, it is necessary to develop a standardized and economical system for introduction and dissemination. However, plant factory is seen as the next growth engines in the short term as they can be used for various purposes, such as overcoming limitations in the agricultural environment, providing stable food supply and isolation from contaminated environments, minimizing waste reduction and building energy.
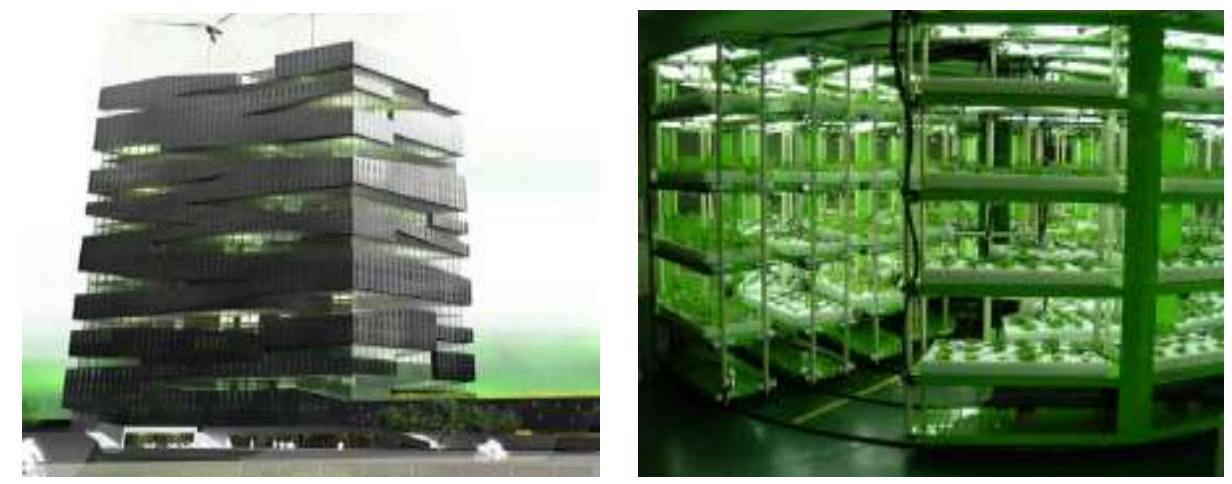

Fig. 1 US Building Plant [2]-[3]

This study develops an automated model of a Plant factory so that self-sufficiency of fresh vegetables without pesticides and chemical fertilizers can be self-sufficient throughout the year. In addition, by utilizing the oxygen generated through the photosynthesis of plants and the carbon dioxide generated by people's breathing, the internal space continuously supplies fresh oxygen free of contaminants to the internal space, and at the same time, the plant factory supplies carbon dioxide for photosynthesis. NASA discovered the plant's air purifying capacity while exploring several ways to purify the air in a fully enclosed spacecraft in space.

Aboard a plant in a completely enclosed spaceship, it was psychologically stable as well as physiological such as fig 1 . According to NASA's findings, about 12 plants were placed in confined spaces containing harmful pollutants, and $80 \%$ of indoor air pollutants such as formaldehyde, benzene and carbon monoxide were removed within 24 hours.

\section{THEORETICAL BACKGROUND}

Generally, a plant factory is a system that automatically and continuously produces a growing environment without being affected by seasons and places by artificially controlling the growing environment under controlled environmental conditions such as light, temperature and humidity, carbon dioxide concentration, and culture medium such as fig. 2. The plant factory has recently been evaluated as a core technology for next generation agriculture in that it is a new growth engine industry that is integrated with BT (Bio technology) and IT (information technology). 


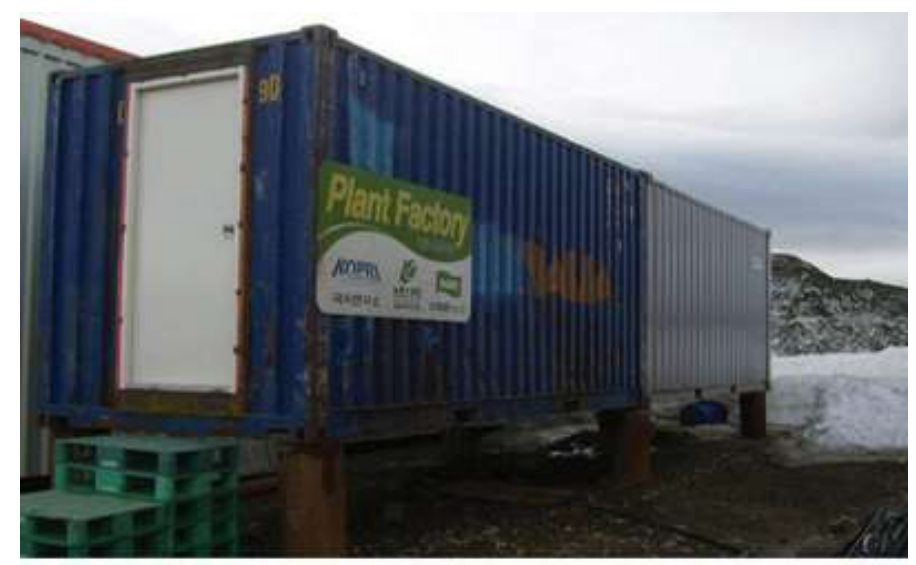

Fig. 2 Examples of plant factory [4]

\subsection{CULTIVATION ENVIRONMENT OF PLANT FACTORY}

Intergrative Environment Control means advanced technical comprehensive control of the environmental factors related to plant growth and worker labor environment, plant factory environment, integrated earth environment and plant growth environment. Most plant factory environmental control means the latter.

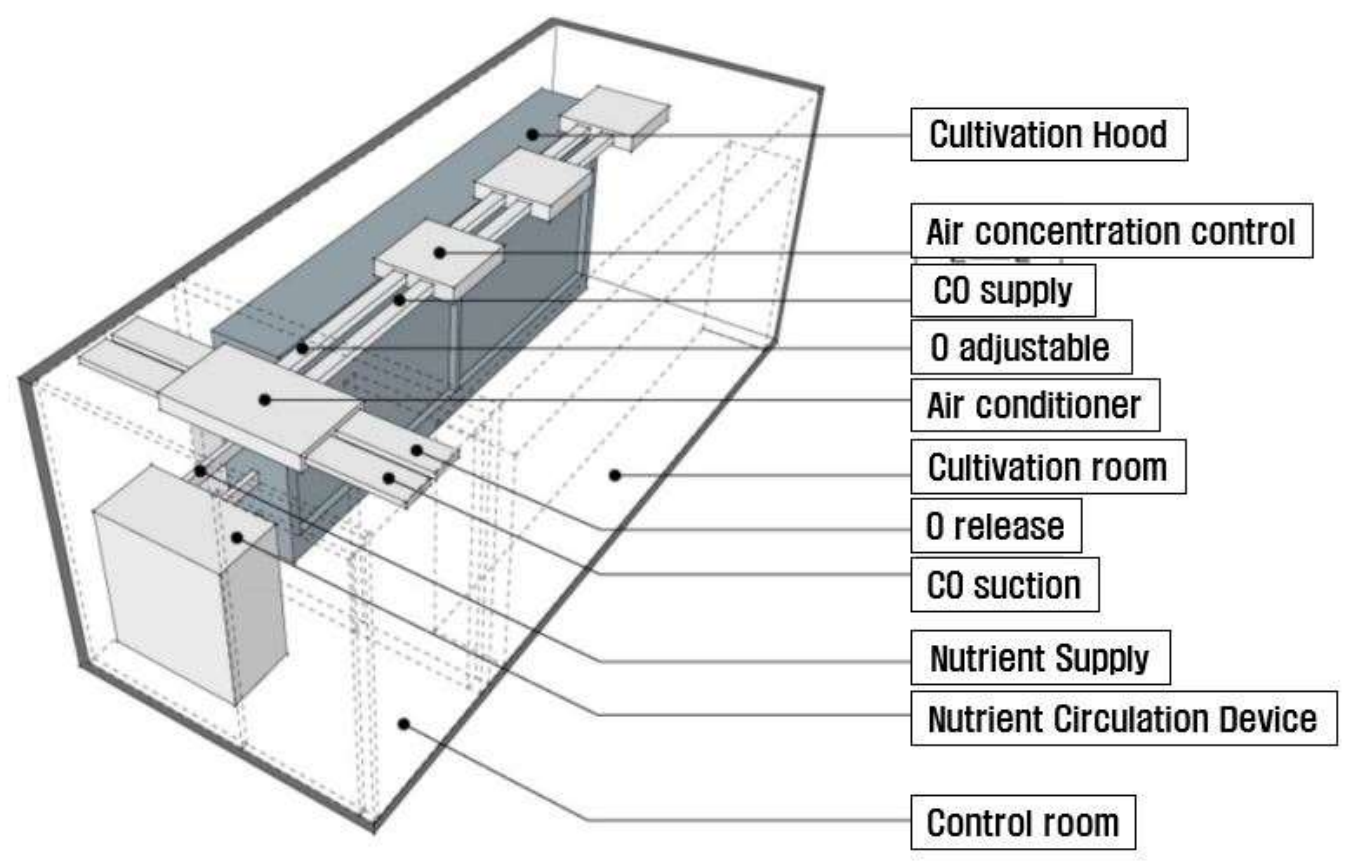

Fig. 3 Basic composition of a plant factory

The main physical environmental factors affecting the photosynthesis rate or growth of plants consist of light intensity, $\mathrm{CO} 2$ concentration, air velocity, air temperature (body temperature), leaf area, air vapor saturation (relative temperature), water potential of plant, and inorganic nutrients. These physical environmental elements are generally integrated and managed for plant cultivation in plant factories of the type shown in fig. 3. For large plant factory, these units can be connected in parallel or in series. To boost the efficiency of the Plant factory, about 2,00 ppm of carbon dioxide is supplied for plant photosynthesis. The light for plant cultivation uses fluorescent lamps and LEDs, and nutrient solution cultivation is used. Nutrient cultivation has a circulation type and a stagnation type, but recently, a circulation type for automatically purifying a chemical 
liquid has been spreading. In addition, it is based on environmental control based on a predictive formula including net photosynthesis rate and growth time derivative [5]-[7].

\section{INTEGRATED CONTROLLER DEVELOPMENT}

\subsection{PLANT FACTORY INTEGRATED SYSTEM ITEMS AND PROCESSES}

Plant factory is a next generation agricultural technology that artificially controls the growth environment (light, air, heat, nutrients) of living organisms in a controlled facility to produce plants like industrial products. However, due to expensive installation and maintenance costs and difficulties in management, the performance is still small. In order to overcome these limitations, it is considered the structure and operation system of the plant suitable for residence characteristics, and propose a convergence system with the residence internal space as a solution to the carbon dioxide concentration control that is not addressed in the existing plant. In particular, in order to make plant management more efficient, integrated automatic control systems should be established [8]. For this purpose, the environmental control factors of plant factory are derived through the light, temperature, moisture, and ventilation control system. In addition, the LED light source and nutrient solution management should be designed to be linked with the automatic control system by applying the water supply and drainage circulation system to smoothly control the plant growth [9].

For the IT-based automatic production control system, a monitoring device for the machine and environment for the production facility and the environment is needed. With these devices, the system must be integrated so that the light source, temperature, etc., associated with plant cultivation can be used smoothly.

\subsection{DEVELOPMENT OF SPATIAL DIVISION MODEL FOR THE REVITALIZATION OF PLANTS AND THE IMPROVEMENT OF OFFICE ENVIRONMENT USING CARBON DIOXIDE BY OFFICE WORKERS}

Factors affecting photosynthesis of plants include light intensity, $\mathrm{CO} 2$ concentration, airflow velocity, temperature and humidity. In order to promote photosynthesis, it is essential to maintain a constant growth environment (temperature $21 \sim 25{ }^{\circ} \mathrm{C}$, relative humidity $70 \sim 80 \%$, wind speed $50 \sim 60 \mathrm{~cm} / \mathrm{sec}$ ). The concentration of carbon dioxide in the air is usually 350ppm, but should be maintained at $1000 \sim 2000 \mathrm{ppm}$ in plant plants. And under the same temperature conditions, maintaining a constant concentration of carbon dioxide rather than the intensity of light becomes a more important factor in plant photosynthesis [10]. If only light is strong at the same carbon dioxide concentration, it promotes leaf dehydration, which in turn hinders plant growth. In the case of using carbon dioxide in the outside air to maintain a proper carbon dioxide concentration in the plant factory, there is a problem that the cultivated plants may be exposed to pollutants in the outside air such as fig. 4-5. Therefore, it is common to maintain a proper concentration of carbon dioxide in a plant factory through a bombe [11].

This phenomenon is increasing the cost burden and management difficulties associated with the purchase of carbon dioxide gas has become a major factor in stabilizing the spread and diffusion of plant plant technology. However, in the space where the clerks live, the concentration of carbon dioxide is instantaneously increased to about $2000 \mathrm{ppm}$ due to the use of breathing, electronic devices, and heating devices, while the oxygen concentration decreases. With this in mind, this study proposes a new coexistence system that fuses offices and plant factories and reverses the roles of carbon dioxide and oxygen. In this study, indoor pollutants and carbon dioxide from office space are discharged to plant factory using temperature and specific gravity difference, and fresh oxygen from plant photosynthesis is supplied to office space again [12]-[13]. 


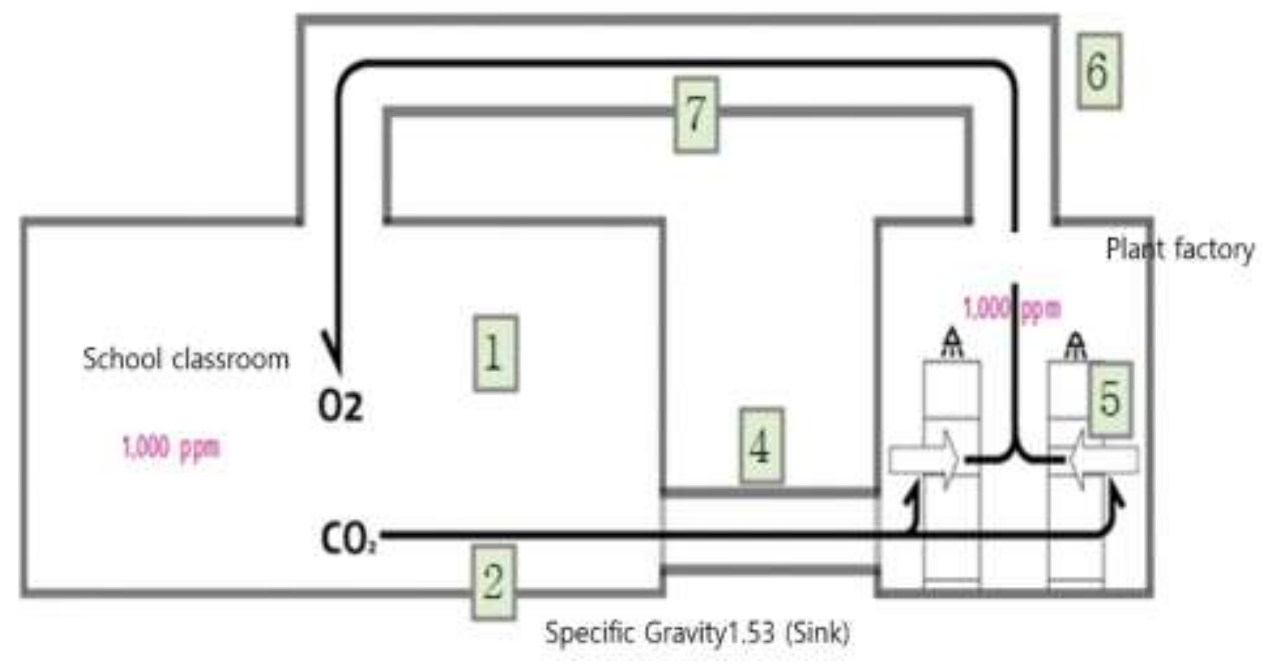

Fig. 4 A Spatial Division Model for the Revitalization of Plants and the Improvement of Office Environment Using Carbon Dioxide by Office Workers

\subsection{DESIGN OF PLANT UNIT MODULE}

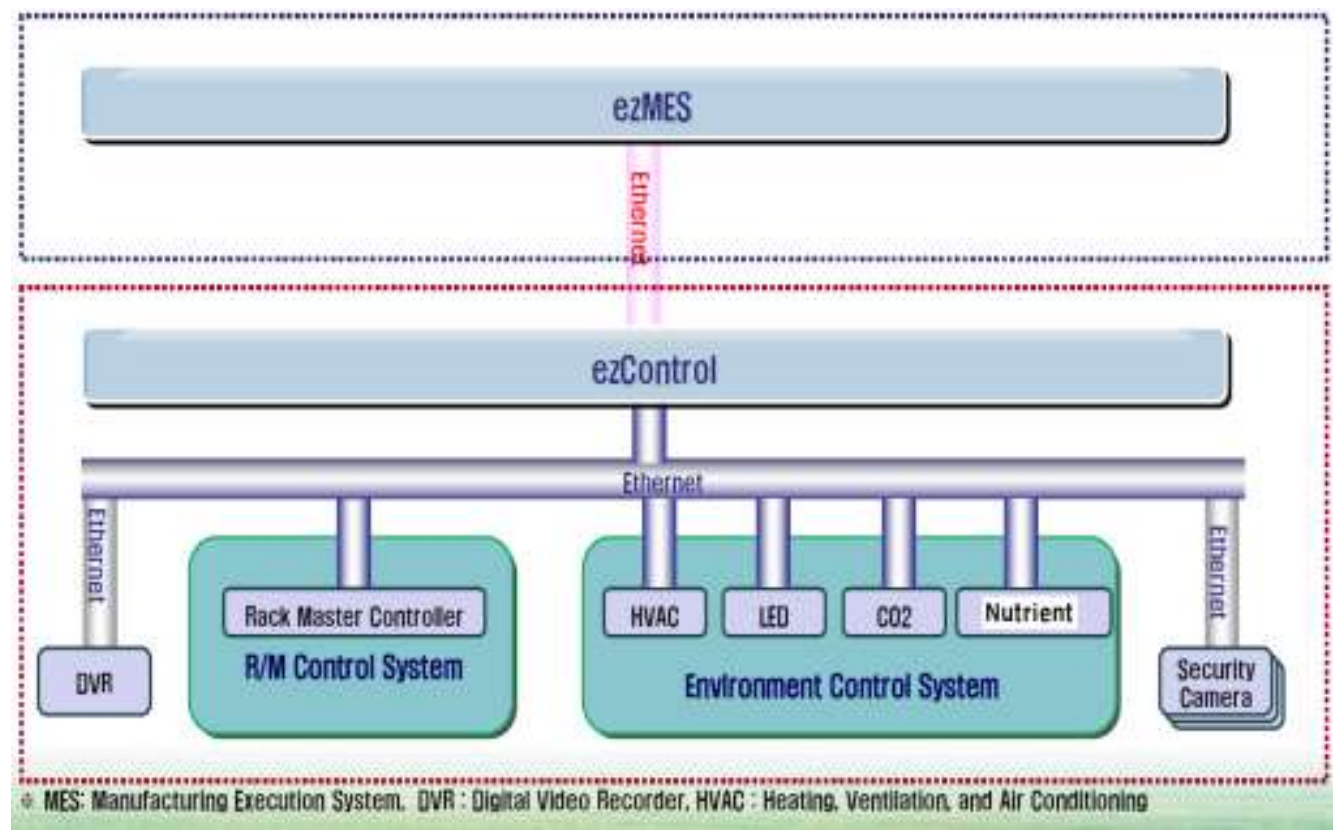

Fig. 5 System Control Diagram

3.3.1. LED LAMP: LED lighting is the most important part of a plant factory, and it is very efficient at converting electricity into light. It has a long lifespan and has all the colored light that sunlight has. In order for plant plants to be economically viable and profitable, the amount of plants produced must be greater than the amount of electricity consumed such as fig.6-7. Only power savings increase the profits and competitiveness of plant plants. Therefore, further research on LED application technology, which is the core technology of plant factory, is needed. For example, a few LEDs were used to apply a few volts of voltage, a few milliamps of 
current, and a few watts of light. However, this study is so extensive that we will focus on the possibility of convergence between residence internal space s and plant factories, except for the scope of the study.

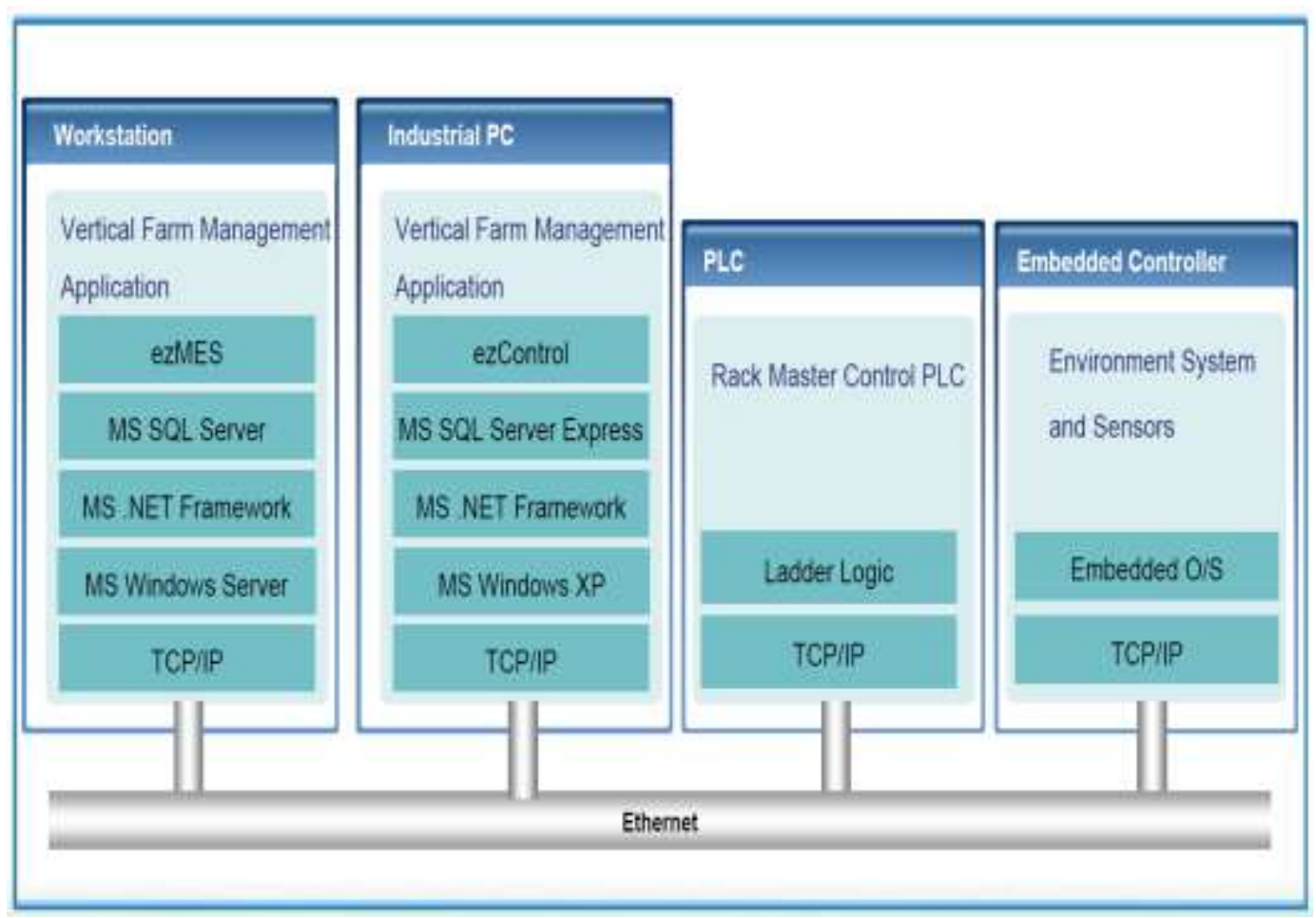

Fig. 6 Software Diagram

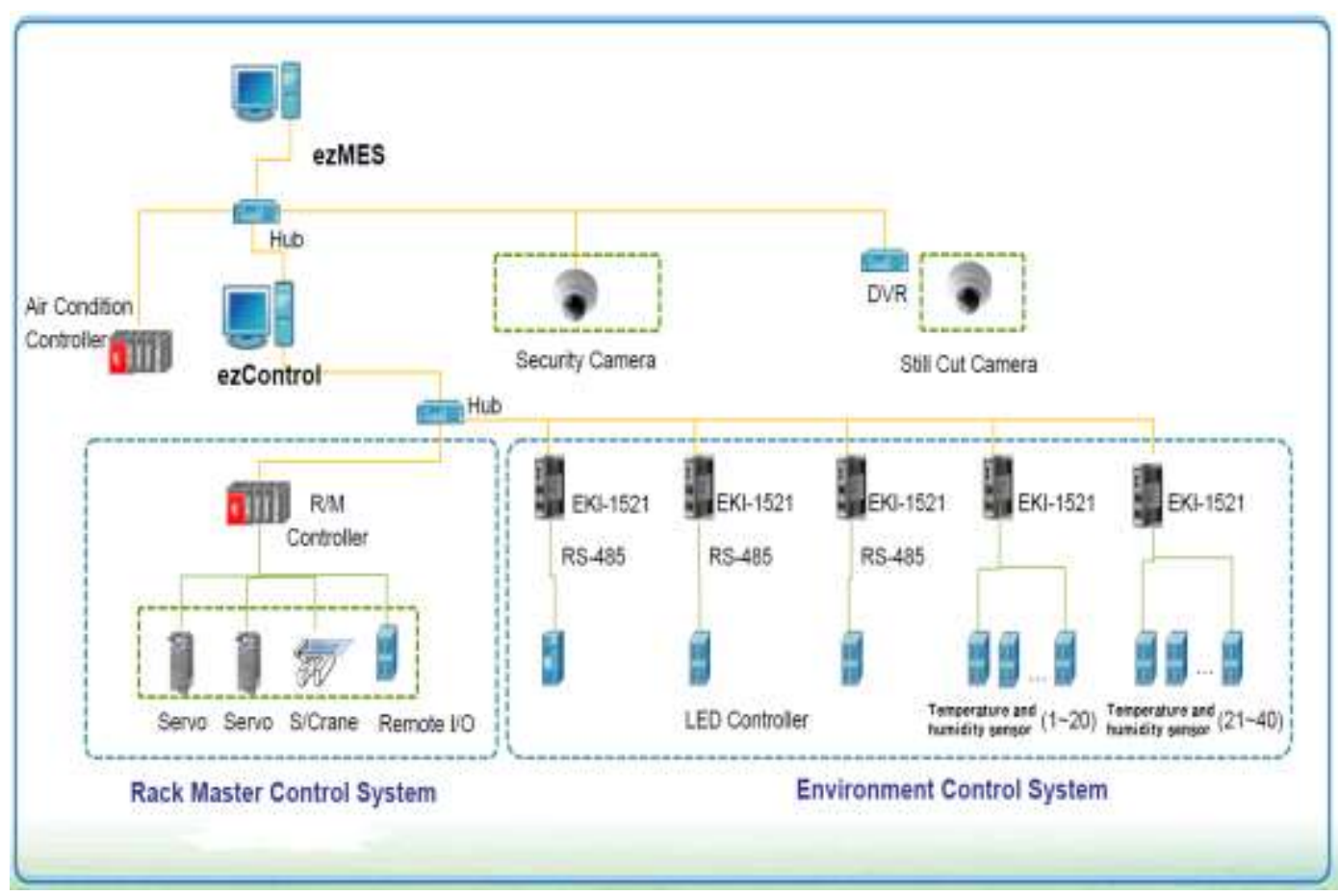

Fig. 7 Network structure

3.3.2. CULTURE SOLUTION: The plant unit module basically uses a circulating hydroponic cultivation system mainly used in container type plant plants. The culture 
solution consists of raw water fertilizer, which is automatically supplied through the pump as needed. In the feeding method, the culture tank is pumped to the highest cultivation tray through a pump and then circulated along the tray to supply nutrient solution to the plants planted in the styrofoam bed. The composition and mixing ratio of these cultures have been generalized by hydroponic experts, and there are many reference materials.

\section{AIR FLOW SIMULATION OF AUTOMATIC CONTROL SYSTEM}

Simulation of the airflow distribution was conducted to verify the performance of the spatial division model for the activation of plant plants and improvement of the office environment using carbon dioxide by the clerks.

For the simulation of the airflow distribution, the Stream program, which is widely used for the analysis of airflow in the architectural engineering environment, was used. The analysis results are as follows. Cases when there is no temperature difference between the internal space and the plant factory and when there is a temperature difference are investigated. To understand the flow of oxygen and carbon dioxide, $\mathrm{CO} 2$ concentration analysis and three-dimensional airflow analysis were performed. The analysis results are as follows.

\subsection{IF THERE IS NO TEMPERATURE DIFFERENCE}

C02 concentration analysis [Stream program]

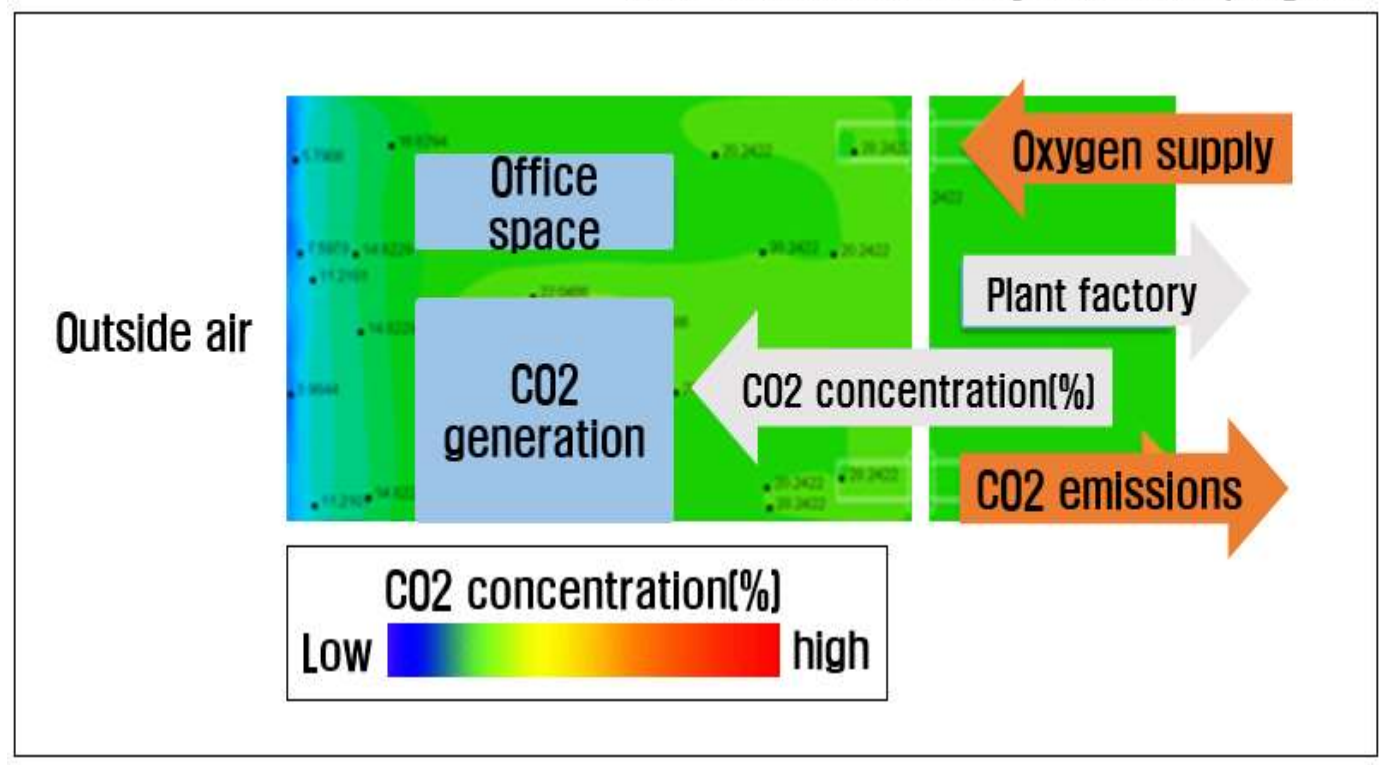

Fig. 8 Carbon dioxide concentration analysis between office space and plant factory when there is no temperature difference

If there is no temperature difference between the interior space and the Plant factory, the air flow is stagnant and the carbon dioxide concentration in the interior space increases such as fig. 8 . 
Airflow Analysis [Stream Program]

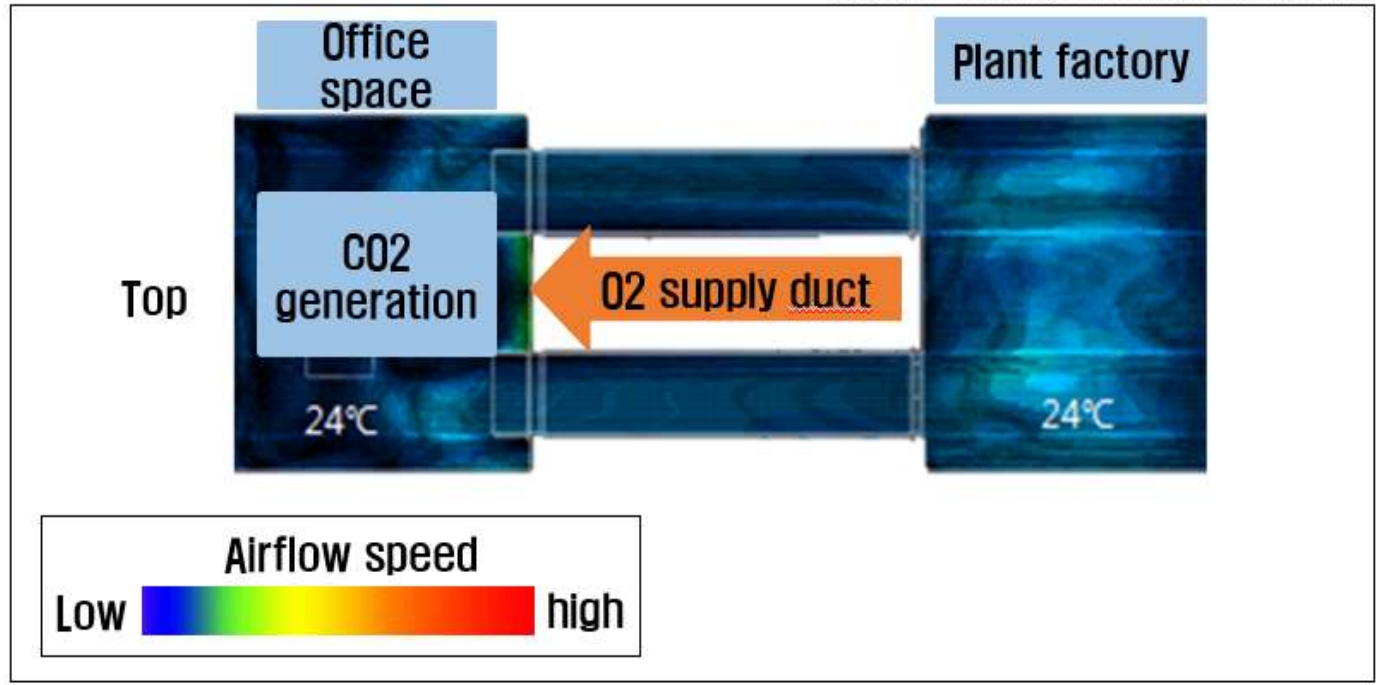

Fig. 9 Air flow analysis between the office space (top) and the plant factory when there is no temperature difference

When the air flow is stagnant, the amount of change in the airflow due to the specific gravity difference between oxygen and carbon dioxide decreases (Upper internal space) such as fig. 9 .

Airflow Analysis [Stream Program]

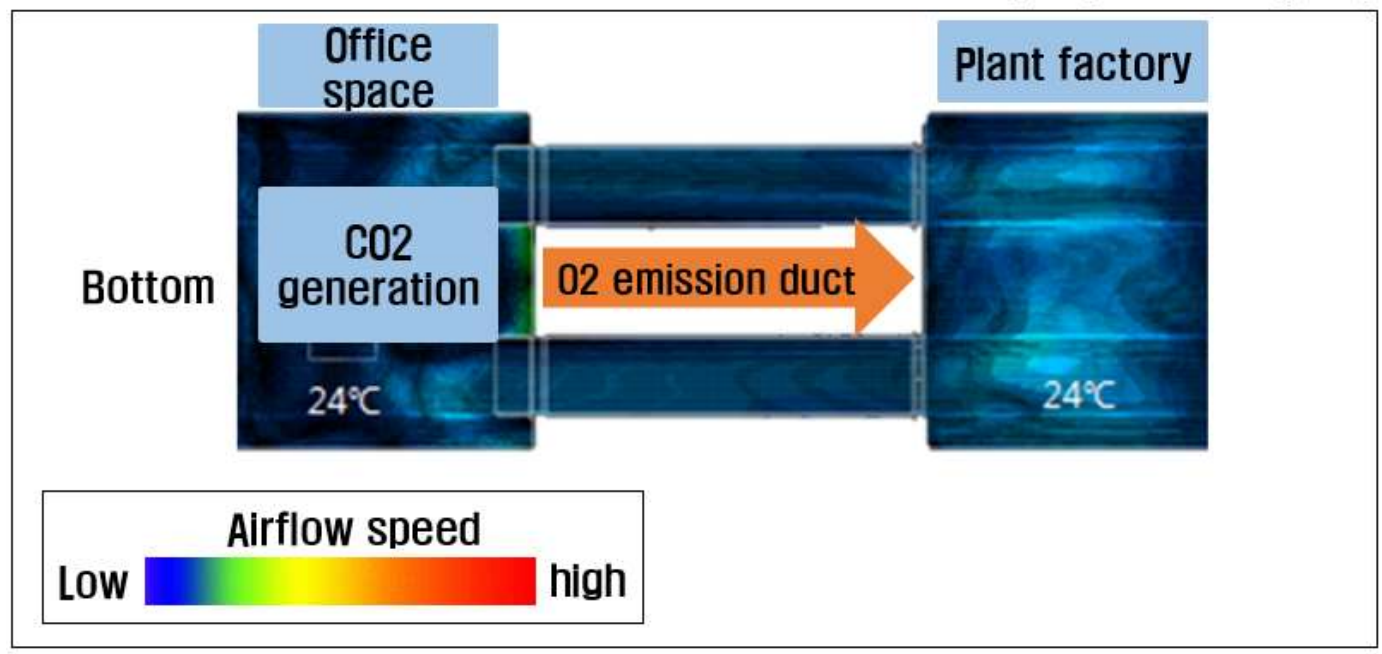

Fig. 10 Air flow analysis between the office space (bottom) and the plant plant when there is no temperature difference

When the air flow is stagnant, the amount of change in airflow due to the specific gravity difference between oxygen and carbon dioxide decreases (Bottom internal space) such as fig. 10. 


\subsection{IF THERE IS A TEMPERATURE DIFFERENCE}

C02 concentration analysis [Stream program]

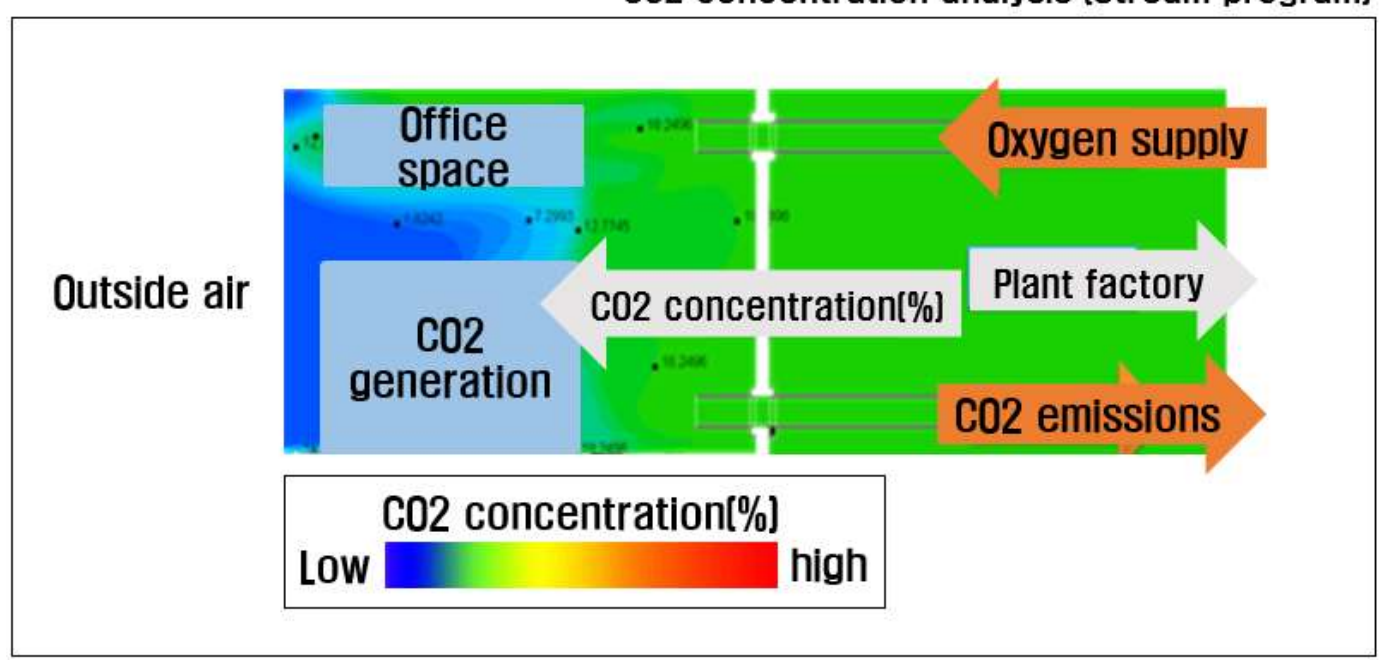

Fig. $11 \mathrm{CO} 2$ concentration analysis between office space and plant factory when there is temperature difference

If there is a temperature difference between the internal space and the plant factory, air flow occurs and the carbon dioxide concentration in the internal space is reduced such as Fig. 11.

Airflow Analysis [Stream Program]

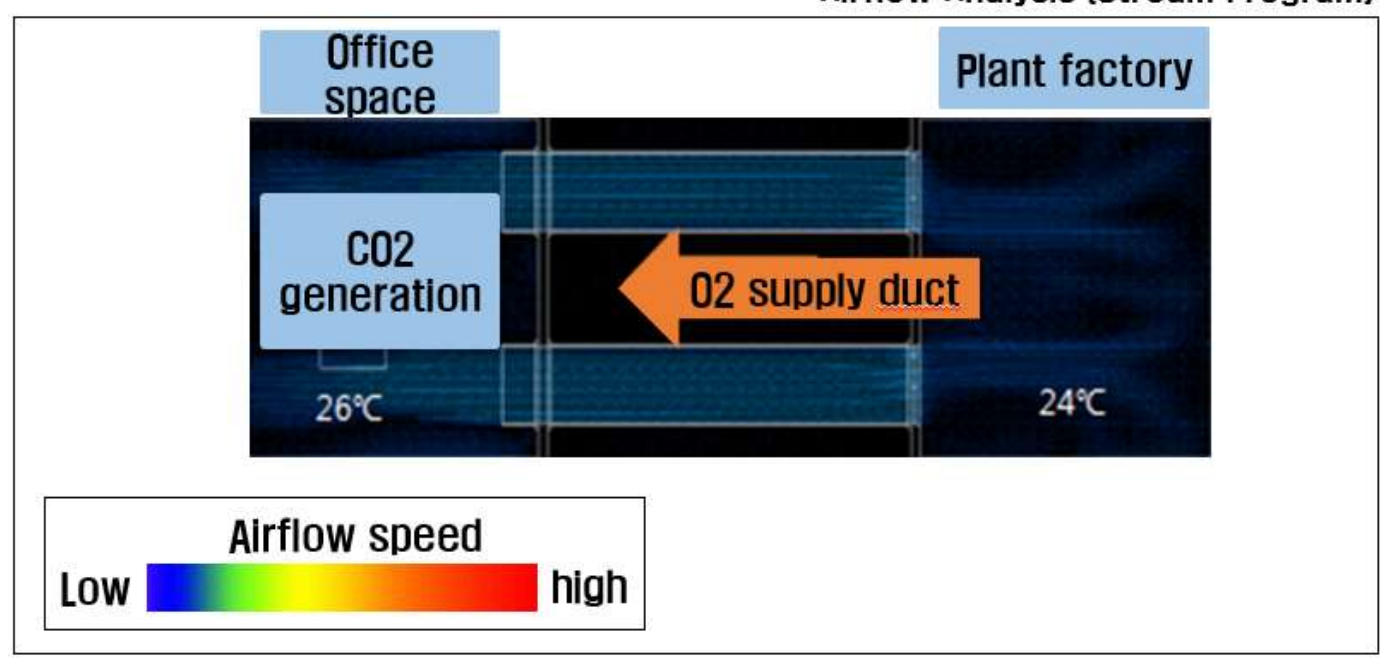

Fig. 12 Air flow analysis between office space (top) and plant plant when temperature difference

When air flow occurs, the amount of change in airflow due to the specific gravity difference between oxygen and carbon dioxide increases. (Upper internal space) such as fig. 12. 


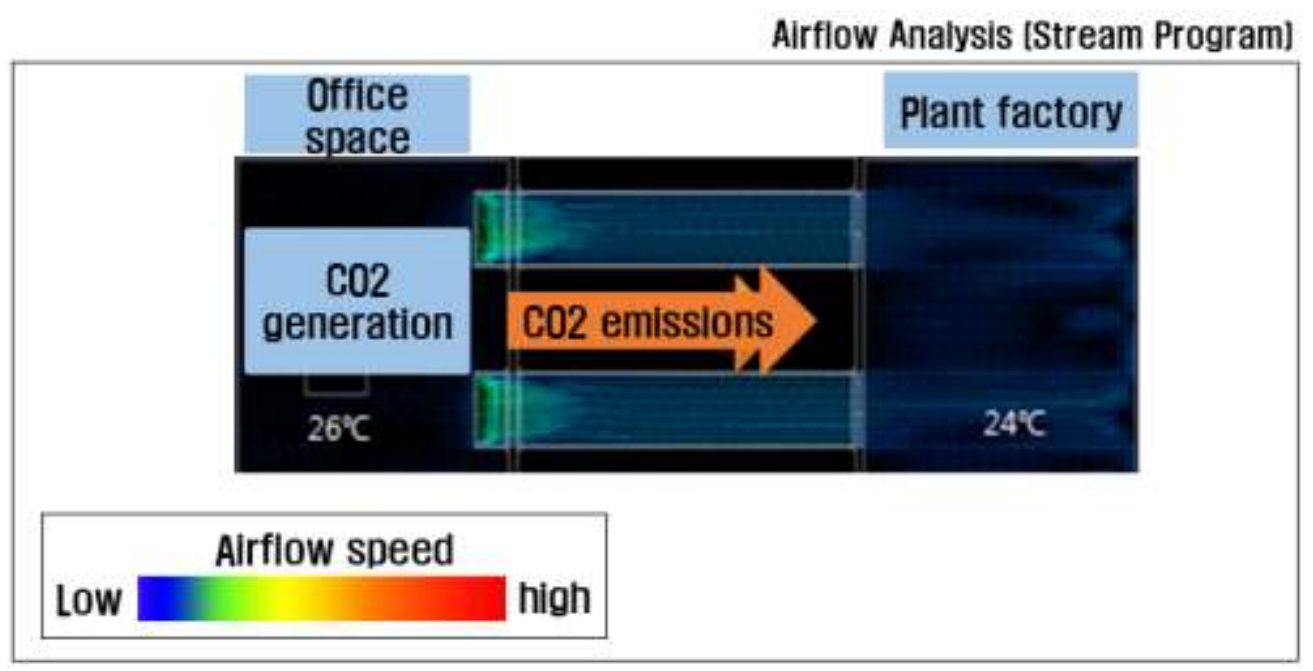

Fig. 13 Air flow analysis between office space (bottom) and plant plant when temperature difference

When air flow occurs, the amount of change in airflow due to the specific gravity difference between oxygen and carbon dioxide increases. (Bottom internal space) such as fig.13.

\section{RESEARCH RESULT ANALYSIS}

\subsection{AIRFLOW SIMULATION OF CONVERGENCE}

For the simulation of the airflow distribution, the Stream program, which is widely used to perform airflow analysis in the civil engineering environment, was used. $\mathrm{CO} 2$ concentration analysis and three-dimensional air flow analysis were conducted to determine the flow of oxygen and carbon dioxide.

\subsubsection{IF THERE IN NO TEMPERATURE DIFFERENCE:}

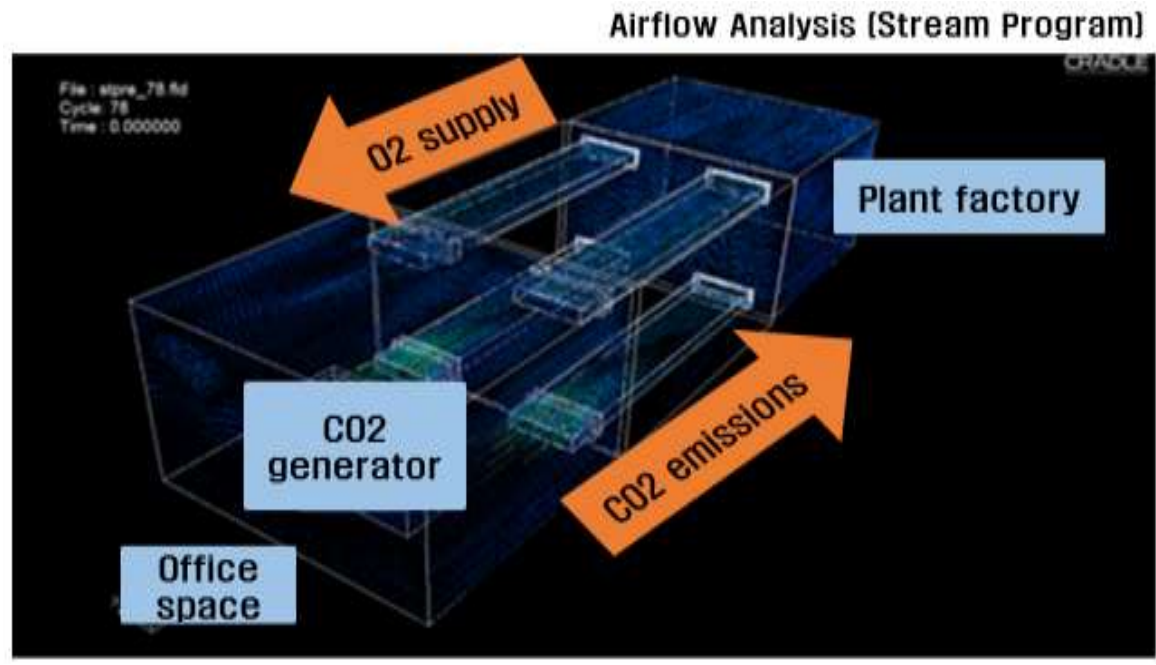

Fig. 14 If there is no temperature difference

If there is no temperature difference between the internal space and the plant factory, the flow of air is stagnant and the carbon dioxide concentration in the internal space increases. When the air flow is stagnant, the amount of change in the airflow due to the 
specific gravity of oxygen and carbon dioxide decreases. When the air flow is stagnant, the amount of change in the airflow due to the specific gravity of oxygen and carbon dioxide decreases. If there is no temperature difference between the internal space and the plant factory, the air flow will be stagnant such as fig. 14 .

5.1.2. IF THERE IS A TEMPERATURE DIFFERENCE: If there is a temperature difference between the internal space and the plant factory, air flow will occur, reducing the $\mathrm{CO} 2$ concentration in the internal space. When the air flow is generated, the amount of change in airflow due to the difference in specific gravity of oxygen and carbon dioxide increases. When the air flow is generated, the amount of change in the airflow due to the difference in specific gravity of oxygen and carbon dioxide increases. If there is a temperature difference between the internal space and the plant factory, air flow is generated and the flow increases due to the specific gravity difference between oxygen and carbon dioxide such as fig. 15 .

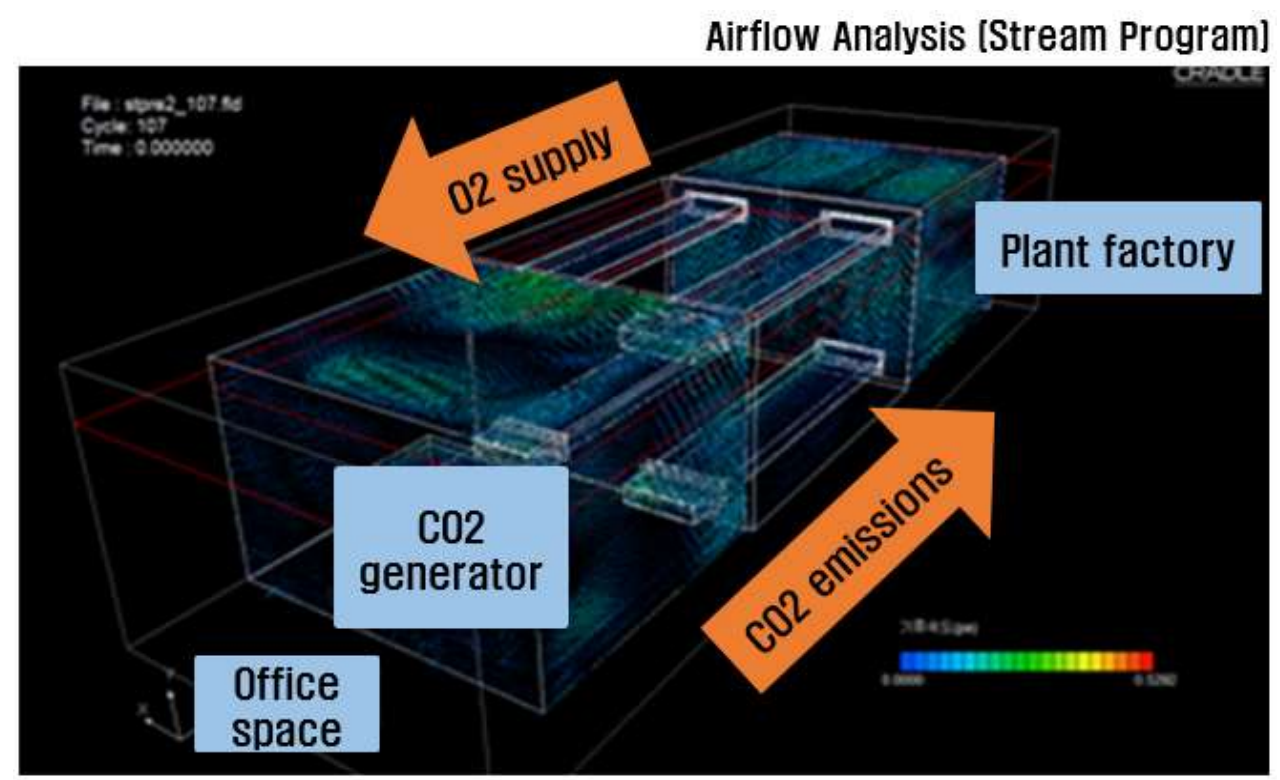

Fig. 15 If there is a temperature difference

\section{CONCLUSIONS}

Currently, plant factories are being introduced in the form of large plant factories and container plant factories, and are constantly developing through the development and systemization of core technologies. The purpose of this study is to develop a plant factory unit that can be efficiently deployed in various residence spaces, but to build an automated program to control the supply of culture medium and the environment of the growing room. This program enables integrated control of LED light, temperature and humidity, $\mathrm{CO} 2$ concentration and ventilation.

Photosynthesis in plant growth in plants is costly and difficult to maintain in proper $\mathrm{CO} 2$ concentrations. In order to overcome these limitations, this study aimed to improve indoor air quality by fusing plant factory with internal living space and actively using photosynthesis of plants. Cases when there is no temperature difference between the internal space and the plant factory and when there is a temperature difference are investigated. To understand the flow of oxygen and carbon dioxide, $\mathrm{CO} 2$ concentration analysis and three-dimensional airflow analysis were performed. The analysis results are as follows. When there is no temperature difference between the internal space and the Plant factory, the air flow is stagnant and the carbon dioxide concentration in the internal 
space increases. In addition, when there is a temperature difference between the internal space and the Plant factory, air flow occurs to reduce the carbon dioxide concentration inside. Once the air flow occurs, the internal carbon dioxide (specific gravity 1.53) is promoted from the bottom to the Plant factory, and the plant factory oxygen (specific gravity 1.1) is promoted from the top to the internal space due to the specific gravity difference. The flow of carbon dioxide and oxygen between the internal space and plant factory can be naturally convected by temperature difference and specific gravity difference, and forced ventilation can also be made by simply using a fan if necessary.

By utilizing the oxygen produced by the photosynthesis of plants and the carbon dioxide produced by people's breathing, fresh oxygen free of contaminants can be supplied to the internal living space, and plants can be supplied with carbon dioxide for photosynthesis. The organic connection between plant factory and residential facilities not only provides a pleasant indoor environment, but also provides economic benefits such as energy savings in buildings, efficient space use, solving food problems and growing all-weather produce.

In order to establish an automatic environmental control system for Plant factory management efficiency, the light (light source, light quantity), temperature, moisture, $\mathrm{CO} 2$ concentration, and ventilation control system should be stabilized in the future in addition, derivation of Plant factory environmental control factors and automatic control test should be conducted. LEDs are more energy efficient, but they produce more heat than fluorescent lamps. [2] Therefore, research is needed to improve the energy saving efficiency by applying the LED cooling device for optimizing the design of energy saving LED light source, developing nutrient solution disinfection system and maximizing LED efficiency. Further research is needed to evaluate the economics of carbon dioxide and IT-based integrated control systems.

\section{REFERENCES}

[1] Hong Jung Hwan, Lee Dong Woon. "A Study on the Intergrative Enviroment Control in Plant Factory. "Proceedings of the Architectural Institute of Korea Conference 31.2 (2017): 69-70.

[2] Chung, Hye-sun. "Plant plant rises in preparation for the food crisis" March 13, 2017, Sejong Economic Daily. sejongeconomy.kr/news/articleView.html?idxno=8615

[3] Cho Hong-seop. "Building Farm, Fruits in the Sky, will be held on January 13, 2009. Hankyoreh Ecology. Ecotopia.hani.co.kr/?document_srl=1387

[4] Chung, Da-hyun. "Declaration of Return on Farms and Plants" September 4, 2011, KAIST Newspaper. times.kaist.ac.kr/news/articleView.html?idxno=1088

[5] Kwang-Young Park, Ji-Hye Jeon and Goo-Man Park, "Integrated Surveillance System and Field Test in Urban Transit System", International Journal of Security and Its Applications, NADIA, ISSN: 17389976 (Print); 2207-9629 (Online), vol.6, no.2, April (2012), pp. 437-442.

[6] C.M. Negi, Amit Pandey, Gireesh G. Soni, Saral K. Gupta and J. Kumar, "Optical CDMA Networks Using Different Detection Techniques and Coding Schemes", International Journal of Future Generation Communication and Networking, NADIA, ISSN: 2233-7857 (Print); 2207-9645 (Online), vol. 4, no. 3, September (2011), pp. 25-34.

[7] Hong Jiwan. "A Study on The Photosynthesis Accelerate by Light Color Composition in Plant Factory. " Journal of the Korean Academic Industrial Society 17(11) (2016): 368-375.

[8] Xueting Niu, "Opportunism Transmit Mechanism Research in Cognitive Network", International Journal of Future Generation Communication and Networking, NADIA, ISSN: 2233-7857 (Print); 2207-9645 (Online), vol. 6, no. 5, October (2013), pp. 1-10.

[9] R., Keenan, M., Miles, I., Butten, M., and Fuenza, G. "Global Foresight Outlook 2007." EFMN, S27-7 (2007): 956

[10] Jeon Hwang-su. "Trends in the development of Auto-IT convergence technology in Korea and abroad. "Korea Multimedia Society Vol. 14 (2009)

[11] Kim Ja Yeon. "research on the actual condition and awareness degree of time shortening. " Korea Institute of Building Construction 10 edition vol.5 (2010): pp.87 95

[12] National IT industry Promotion Agency. "Construction and IT convergence technologies Roadmap. " Technology Trends Weeks Vol. 1390 (2009)

[13] Manoj Badoni and Sunil Semwal, "Discrete Distance and Water Pit Indicator using AVR ATmega8 in Electronic Travel Aid for Blind", International Journal of Disaster Recovery and Business Continuity, NADIA, ISSN: 2005-4289 (Print); 2207-6425 (Online), vol.2, November (2011), pp. 1-8. 